\title{
Tool Wear Analysis on Five-Axis Flank Milling for Curved Shape Part - Full Flute and Ground Shank End Mill
}

\author{
Sundi Syahrul Azwan ${ }^{1, *}$, Muhamad Mohd Razali ${ }^{2}$, Kasim Mohd Shahir ${ }^{2}$, and R.Abdullah \\ R. Izamshah ${ }^{2}$ \\ ${ }^{1}$ Faculty of Engineering Technology, Universiti Teknikal Malaysia Melaka, Hang Tuah Jaya, 76100 \\ Durian Tunggal, Melaka, Malaysia \\ ${ }^{2}$ Faculty of Manufacturing Engineering, Universiti Teknikal Malaysia Melaka, Hang Tuah Jaya, \\ 76100 Durian Tunggal, Melaka, Malaysia
}

\begin{abstract}
This paper is a study on full flute (extra-long tool) and ground shank end mill wear analysis by utilizing five-axis CNC to implement flank milling strategy on curved shape part. Five-axis machining eases the user to implement variations of strategy such as flank milling. Flank milling is different from point milling. Point milling cuts materials by using the tip of the tool whereas the flank milling uses the cutting tool body to cut material. The type of cutting tool used was end mill $10 \mathrm{~mm}$ diameter with High Speed Steel (HSS) material. One factor at a time was utilized to analyze the overall data. Feed rate and spindle speed were the two main factors that been set up equally for both full flute and ground shank end mill. At the end of this research, the qualitative analysis based on tool wear between full flute and ground shank end mill is observed. Generally, both types of cutting tools showed almost the same failure indication such as broken edge or chipped off edge, formation of pinned hole on the surface and serration formation or built-up edge (BUE) on the primary flute. However, the results obtained from the enlarged images which were captured by Optical Microscope indicated that, the ground shank end mill is better than the full flute end mill.
\end{abstract}

\section{Introduction}

There are a lot of parts that are difficult to machine especially in industry mentioned as aerospace, molds and dies, automotive, and heavy machinery. Usually, these industries need to produce complex structure and hard materials. Therefore, it is extremely need an analysis on tool wear. Most of the studies are concentrating on the strategy of machining such as tool path algorithm, ruled surface, and cutting stability to reduce tool wear while do machining. All these research are good to be implemented but the study on tool wear need to be taken into account before it. Hou, Y. [1] stated that the tool wear is a key factor

* Corresponding author: syahrul.azwan@utem.edu.my 
affecting the machining quality. They also agreed that the need for tool wear analysis is important in the machining. In the previous studies, Choudhury and Rath [2] proposed a milling tool flank wear assessment approach based on the relationship of the average cutting force coefficients and the tool flank wear. Sarhan [3] et al. proposed an approach for tool wear detection based on the variation of cutting force components in frequency domain. Bhattacharyya [4] et al. used the average signal energy and signal mean square deviation as the tool wear state indicators for the single-tooth milling in same conditions. Kuljanic and Sortino [5] defined the normalized cutting force (NCF) indicator and the torque-force distance (TFD) indicator by cutting forces, and characterized the tool wear state through these two indicators. In this research, tool wear analysis on flank milling was done and it slightly different from the others studies. This research analysis approaches the qualitative measurement of the tool named full flute and ground shank end mill after the machining by observing the conditions. Basically, full flute is extra-long (long series) tool which is very useful in machining deeper contour or profile where is not accessible by normal or standard series of tool. In the other hand, ground shank end mill is a normal or typical series cutting tool with standard flute length. There is minor adjustment made to the shank diameter by grinding out a few millimeters to ensure that the shank will not be rubbing up the work piece while machining.

\section{Methodology}

This research activity started with preparation of the part modelling which was assisted by Computer Aided Design (CAD) software. The modified part modelling was then gone through a process called machining programming which was assisted by Computer Aided Manufacturing (CAM) software. The completed program was post processed and transferred to the Computer Numerical Control (CNC) machine center to perform the actual machining. Fig. 1 illustrates the CAD model with different view point namely as isometric, top, and side views.
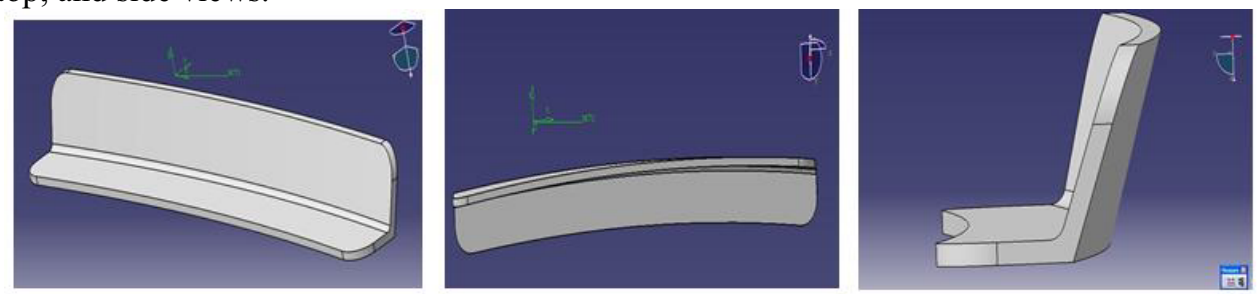

Fig. 1. Part or CAD model selected in isometric, top and side views.

At the present moment, not every industry awares of the advantages of using ground shank tool especially in aerospace industry. In general, ground shank tool is very useful in cutting at the deeper and shallow area. There are number of methods available in grinding the tools' shank. In shank grinding process, the even roundness diameter is the most critical criteria in order to ensure the balancing and stability of the tool during the machining. In machining, the stability and the balancing of the tool plays a very important role in giving the best machining result especially the surface roughness and the dimensional accuracy. Imbalanced motion can create high chattering and vibration phenomenon. Ground shank tool can be seen clearly as shown in Fig. 2 below. The reachable flute length for both tool was differentiated by $10 \mathrm{~mm}$. Feed rate and spindle speed remained constant for both physical machining.

Optical Microscope was utilized to observe the condition of both tools closely. The magnifying power used to analyze the tool wear images in this study was 3500 times. The 
accuracy of the measurement taken is relying on the user's skill to plot and perform the manual measurement using the tools provided. In this research the tool wear analysis was categorized into a few areas namely front face of the flutes, side or body of the tool and slot area or secondary flutes. For the side or the body of the tool, there were another three other different sections were taken into consideration such as bottom, middle and top portion. In general, the analysis done was only concentrated on physical comparison. The discussion and conclusion made were based on the overall appearance and physical images of the tool's sections only.

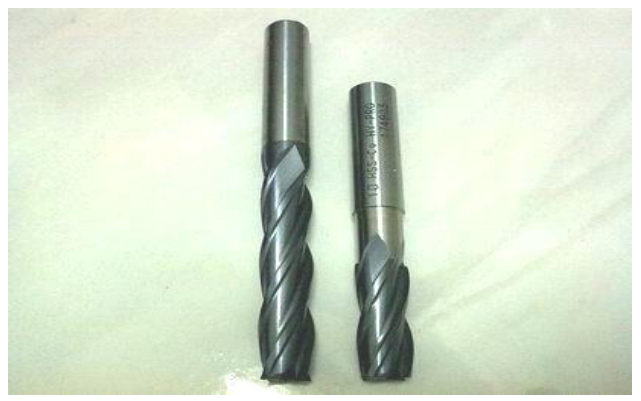

Fig. 2. The physical comparison of the long series/full flute and ground shank end mill.

\section{Result and discussion}

At the end of the machining, the analysis on tool wear is thoroughly observed. For this research, the tool wear of the tools were investigated by looking at the physical appearance of the tool using the Optical Microscope. The wear of the tool was only observed by looking at the overall tool's geometry captured without any specific physical measurement taken. An interpretation was made from the differences observed to the both end mills. In other words, the approach of analyzing the tool wear in this research was qualitative approach or first level interpretation analysis. In order to obtain better overview on which geometry of cutting tool shall resulting better outcomes in tool wear, a fair comparison needs to be done.

Therefore, both setups were having the same parameters. The spindle speed applied was $4500 \mathrm{rpm}$ whilst $1000 \mathrm{~mm} / \mathrm{min}$ for the feed rate. There were three specimens or machined part examples machined for every type of cutting tool. The most significant result obtained was the chipped off edges. It happened in full flute but not in ground shank end mill which clearly indicated by Fig. 3. In addition, there were much more serration or built up edge (BUE) formed and pinned holes tabulation were higher and bigger in size for the full flute end mill to be compared to the ground shank tool as illustrated in Fig. 4.

This failures happened were probably because of high cutting forces occurred during machining. The full flutes end mill has longer cutting flute length which encourages the creation of high vibration during machining [1]. On the other hand, there was different situation happened for ground shank tool. Since the amount of the contact area with the material was less, this automatically reduces the tendency of vibration occurrence. The main tool failure mode was catastrophic chipping of the cutting edge for all cutting operations. In five-axis milling, the tool life would be expected to increase due to the variation of the contact area [2]. Probably bull nose or radius type end mill can be considered in replacing the flat end mill in order to overcome this chipped off edges problem in machining of the curved shape profile. 

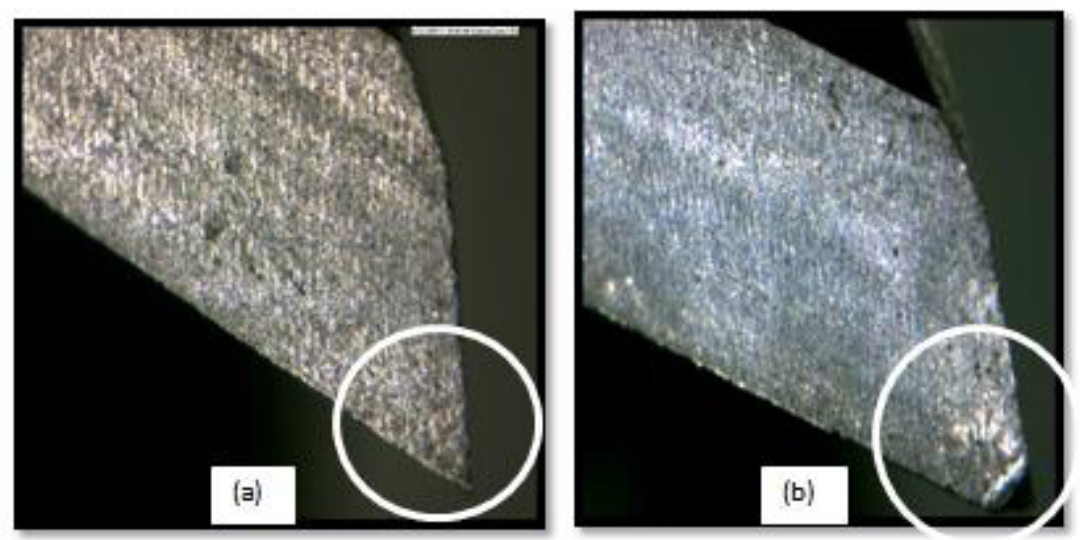

Fig. 3. Comparison of chipped off edges between (a) ground shank and (b) full flute end mill.
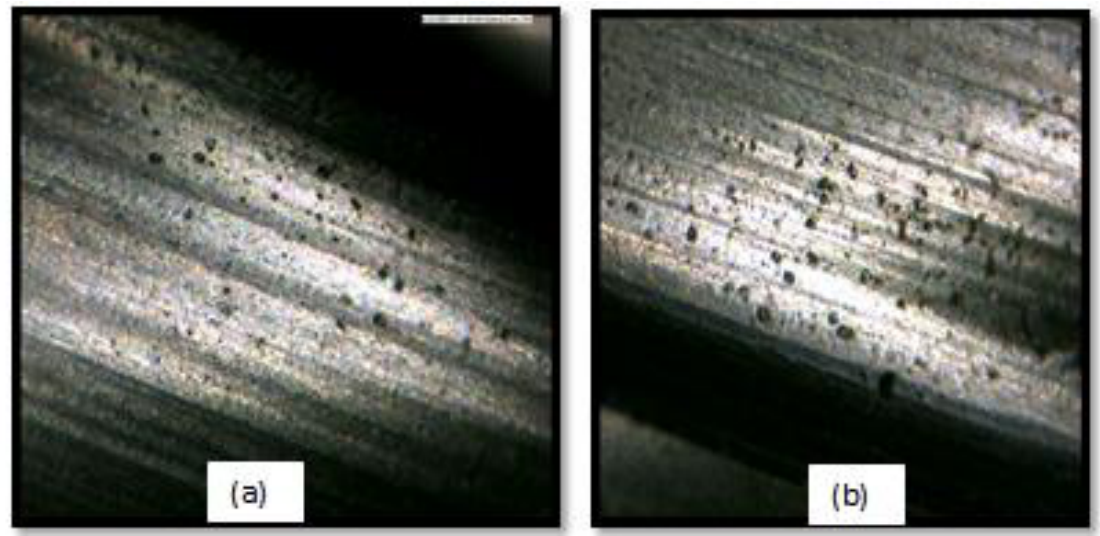

Fig 4. Comparison of pinned holes formation between (a) ground shank and (b) full flute end mill.

\section{Conclusion}

As for the conclusion, both types of cutting tools showed almost the same failure indication such as broken edge or chipped off edge, formation of pinned holes on the surface and serration formation or built-up edge (BUE) on the primary flute. However, the results obtained from the ground shank end mill was better than the full flute end mill. In the specific comparison made, the ground shank resulted no chipped off edges with minimum pinned holes and built-up edge (BUE) formation at constant machining parameters. Ultimately, in flank cutting of curved shape profile the ground shank end mills is preferably to be used rather than full flute end mill as it is more economical.

Author acknowledges, with gratitude, authors' debt of thanks to Universiti Teknikal Malaysia Melaka (UTeM) for having sufficient facilities and equipment along this study was conducted. Special thanks to the funding body; Ministry of Higher Education Malaysia for the research grant (RAGS/1/2014/TK01/UTEM/B00079) that support most of the involved cost to complete this study. 


\section{References}

1. Y. Altintas, S. Engin, E. Budak, Journal of Manufacturing Science and Engineering, 121 (1999)

2. R. Sarhan, A. Sayed, A. Nassr, R. M. El Zahry, J. Mater. Process. Tech. J., 109, (2001)

3. C.E. Becze, P. Clayton, L. Chen, T.I. El-Wardany, M.A. Elbestawi, International Journal of Machine Tools \& Manufacture, 40 (2000)

4. E. Kuljanic, M. Sortino, Int. J. Mach. Tool. Manu. J., 45 (2005)

5. I. Korkut, M. A. Donertas, The influence of feed rate and cutting speed on the cutting forces, surface roughness and tool-chip contact length during face milling, (2007)

6. P. Bhattacharyya, D. Sengupta, S. Mukhopadhyay, Mech. Syst. Signal Process, 21 (2007).

7. F.H. Ramy, G. Hu, B. Alain, Comput. Aided Des., 45 (2013)

8. S. K. Choudhury and S. Rath, J. Mater. Process. Tech. J., 99 (2000).

9. S.A. Sundi, M.R. Muhamad, B.A. Bakar, R.I.R. Abdullah, Science International (Lahore), 26 (2014)

10. Y. Hou, D. Zhang, M. Luo, B. Wu, 2014 IEEE/ASME International Conference on Advanced Intelligent Mechatronics, (2014) 